\title{
GESTÃO INTERNACIONAL E CULTURA NACIONAL BRASILEIRA: retratos das abordagens e pontos de contato
}

\author{
International management and brazilian national culture: \\ approaches and contact points
}

\author{
Carlos Roberto Domingues ${ }^{a}$, Janaína Maria Bueno ${ }^{\mathrm{b}}$ \\ a Doutorando em Administração de Empresas, EAESP-FGV/SP, São Paulo, SP - Brasil, e-mail: carlosdomingues@gvmail.br \\ b Doutoranda em Administração de Empresas, EAESP-FGV/SP, Faculdades Integradas do Brasil-UNIBRASIL, Curitiba, PR \\ - Brasil, e-mail: janainab@unibrasil.com.br
}

\begin{abstract}
Resumo
Este artigo investiga as abordagens e os pontos de contato entre a gestão internacional e a cultura nacional, no contexto organizacional brasileiro. Para tanto, foi feita uma pesquisa documental em artigos sobre gestão internacional publicados em anais de congressos e periódicos brasileiros dos últimos seis anos (2001 a 2006). Como resultado foi identificado que os artigos podem ser separados em dois grupos, o que contém maior número de artigos não aborda a questão da cultura brasileira, prestandose a tratar elementos inerentes a testes e aplicação de teorias e modelos de estratégias e gerenciais. Já no segundo grupo, composto por uma minoria de artigos, foi percebido preocupação com a questão da cultura. Nestes artigos às características como a ambigüidade e a facilidade na adoção de modelos e práticas de gestão são evidenciadas. Também foram identificadas considerações a respeito de traços como a descontração, a camaradagem, a tendência em personalizar as relações de trabalho, a facilidade de adaptação, a flexibilidade e a paciência. Destacam-se a complexidade da cultura brasileira e a percepção de diferenças culturais entre suas regiões, o que caracteriza a existência de pontos de contato entre a Gestão Internacional e a Cultura Nacional Brasileira.
\end{abstract}

Palavras-chave: Gestão internacional; Cultura nacional; Estratégia; Diferenças culturais.

\section{Abstract}

This article researches the approaches and contact points between international management and national cultural, inside Brazilian organizational context. A documental research was done international management articles published in the Brazilian academy meetings and Brazilian journals since last six years (2001 from 2006). The results showed two different groups of articles: one of them contains the most number of articles and it doesn't treat Brazilian culture 
issues, it treats elements of tests and theory applications and strategy models. The second group, it is composed by minority number of articles, concerns about culture issues. In these articles, characteristics like ambiguity and facility to adopt management models and practices are shown up. It was found considerations about characteristics like informality, the camaraderie, and the tendency in personalize the relations of work, the facility of adaptation, the flexibility and the patience. The Brazilian culture complexity and individual perceptions of cultural differences among regions are detached. This fact characterizes the existence of contact points between International Management and Brazilian Culture.

Keywords: International management; National culture; Strategy; Cultural differences.

\section{INTRODUÇÃO}

A globalização como um fenômeno emergente e ainda em construção pode ser entendido como um processo de internacionalização das trocas sejam estas de produtos, serviços ou conhecimento. Para fazer parte desta dinâmica, as organizações precisam operar com perspectiva global e se adaptar às diferentes demandas de seus stakeholders.

Ademais, existem poucas organizações nas quais a estrutura de poder está concentrada em uma única pessoa ou único grupo, com uma cultura uniforme permeando toda a organização. Em grande parte, as organizações são multiculturais e possuem vários centros de poder. Sendo assim, qualquer mudança ou decisão poderá provocar uma multiplicidade de reações tanto positivas quanto negativas frente às suas estratégias.

Por isso, é preciso analisar os pressupostos de cultura nacional que afetam o ambiente organizacional. Os pressupostos básicos, os costumes, as crenças e os valores, bem como os artefatos que caracterizam a cultura de uma empresa, trazem sempre, de alguma forma, a marca de seus correspondentes na cultura nacional. Não há como, portanto, estudar a cultura das organizações que estão inseridas numa sociedade, sem estudar a cultura - ou as culturas - dessa sociedade.

Ao olharmos para a realidade organizacional brasileira, em um primeiro momento, podemos ter a impressão de estarmos presenciando fenômenos gerenciais típicos, muito parecidos com quaisquer outros observados em vários lugares do mundo. Porém, é fundamental um olhar mais profundo dos traços elementares da cultura nacional para entender os fenômenos por trás da fachada. A textura sócio-cultural brasileira é complexa e multifacetada, influenciando as organizações e a forma como são geridas.

Assim, este estudo pretende levantar dentro da literatura sobre Gestão Internacional os trabalhos que consideram a perspectiva cultural brasileira e analisar as suas contribuições para a compreensão da nossa realidade organizacional no cenário de internacionalização das organizações.

\section{A gestão internacional}

O comércio internacional não é uma realidade nova. Desde a Antiguidade, diferentes povos praticavam formas de comercialização entre si. Porém, determinados períodos históricos registraram explosões dessa comercialização, extrapolando o que até então era praticado. Um desses momentos foi o período de descobrimento de novas rotas marítimas para o comércio de especiarias e a chegada dos europeus à América. As nações responsáveis pelo transporte e comercialização destes produtos passaram a dominar o mercado da época. Nota-se, que nesse movimento, mais foram os produtos que se internacionalizaram que propriamente dito as organizações.

A mais nova explosão no comércio internacional se deu com a abertura de mercados e a expansão dos negócios que ocorreu, principal- 
mente, a partir da década de 70 e fortaleceu-se na década de 90. Este movimento é, para Ortiz (2003, p. 15), um "fenômeno emergente", um processo ainda em construção e é denominado globalização. Principalmente se o entendimento de globalização estiver de acordo com o sentido de "internacionalização das trocas, de produtos e de conhecimento". A globalização caracteriza-se por uma busca intensa por novos mercados, pela alta competitividade entre os fornecedores e por clientes cada vez mais exigentes e cientes da sua força.

Um fator que muito contribuiu para este fenômeno foi o rápido avanço tecnológico. A distância geográfica, outrora barreira à expansão dos negócios, foi radicalmente diminuída com a consolidação da tecnologia de informação e das telecomunicações. De outro lado, as pressões competitivas, a flexibilidade de trabalho e o enfraquecimento da mão-de-obra sindicalizada levaram à redução de despesas com o Estado do bem-estar social, que era o alicerce do contrato social na era industrial. Como argumenta, Castells ( 2000, p. 413):

[...] as novas tecnologias da informação desempenharam papel decisivo ao facilitarem o surgimento desse capitalismo flexível e rejuvenescido, proporcionando ferramentas para a formação de redes, comunicação à distância, armazenamento/processamento de informação, individualização coordenada do trabalho e concentração e descentralização simultâneas do processo decisório.

Nesse cenário, as organizações fazem seus movimentos de internacionalização e criam estruturas fora de seus países de origem. Desta forma, neste momento, são as empresas e não somente seus produtos que atravessam as fronteiras. Para Bartlett e Ghoshal (1992, p. 3), as pressões transformaram o jogo da competitividade global, forçando as organizações a repensarem seus métodos tradicionais de estratégia mundial. As novas estratégias, por sua vez, "levantaram questões sobre a adequação das estruturas e processos organizacionais usados no gerenciamento das operações mundiais."
Em diferentes indústrias, as organizações desenvolveram respostas estratégicas e estruturais muito diferentes para as mudanças que estão ocorrendo em seu ambiente. Para Bartlett e Ghoshal (1992, p. 4), poucas organizações mundiais entraram na década de 80 com o tipo de estrutura que poderia responder de modo eficiente ao novo ambiente de atividades. As tendências que impulsionam esta mudança no jogo competitivo derivam-se das forças de globalização e localização. O custo de pesquisa e desenvolvimento, juntamente com a diminuição dos ciclos de vida das novas tecnologias e dos produtos por elas gerados, levou as organizações a buscar um volume global a fim de amortizar, o mais rápido possível, este pesado investimento.

Neste processo, os estudos sobre a gestão de empresas voltaram-se para um novo campo: a gestão internacional. Seja por meio de uma abordagem mais ampla de estratégias de internacionalização, seja tratando mais especificadamente áreas funcionais (como marketing internacional, gestão de pessoas, finanças, entre outras) ou ainda unindo as duas perspectivas (estratégia e áreas funcionais), a literatura sobre gestão internacional se configura como crescente e em processo de consolidação de conhecimentos.

Dentre os vários aspectos explorados, podemos destacar os estudos sobre a internacionalização de empresas que, segundo Ortiz (2003, p. 15), é vista como aumento da extensão geográfica das atividades econômicas por meio das fronteiras nacionais. Segundo Caldas e Wood Jr. (1999, p. 407), a internacionalização de uma organização implica "a expansão de interfaces entre a empresa e as nações, às vezes levando à invasão ou dominação política". Os autores Bartlett e Goshal (1992) defendem a idéia de que a internacionalização é um processo que pode ocorrer de formas diferentes, dependendo do tipo de organização, tipo de produto e estratégia de internacionalização. A Tabela 1 agrupa os quatro conceitos usados por estes autores para definir a organização conforme seu processo de internacionalização: 
TABELA 1 - Características organizacionais

\begin{tabular}{lllll}
\hline $\begin{array}{l}\text { Característica } \\
\text { Organizacional }\end{array}$ & Multinacional & Global & Internacional & Transnacional \\
\hline $\begin{array}{l}\text { Configuração de } \\
\text { ativos e recursos }\end{array}$ & $\begin{array}{l}\text { Descentralizada e } \\
\text { auto-suficiente } \\
\text { em nível nacional }\end{array}$ & $\begin{array}{l}\text { Centralizada e } \\
\text { escala global }\end{array}$ & $\begin{array}{l}\text { Fontes de } \\
\text { competências } \\
\text { básicas } \\
\text { centralizadas, } \\
\text { outras descentralizadas }\end{array}$ & $\begin{array}{l}\text { Dispersa, } \\
\text { interdependente } \\
\text { e especializada }\end{array}$ \\
$\begin{array}{l}\text { Papel das } \\
\text { subsidiárias no } \\
\text { exterior }\end{array}$ & $\begin{array}{l}\text { Sentir e explorar as } \\
\text { oportunidades locais }\end{array}$ & $\begin{array}{l}\text { Implementar } \\
\text { estratégias } \\
\text { da companhia-mãe }\end{array}$ & $\begin{array}{l}\text { Adaptar ealavancar } \\
\text { competências da } \\
\text { companhia-mãe }\end{array}$ & $\begin{array}{l}\text { Contribuições } \\
\text { diferenciadas das } \\
\text { unidades nacionais a } \\
\text { operações mundiais } \\
\text { integradas }\end{array}$ \\
$\begin{array}{l}\text { Desenvolvimento } \\
\text { e difusão do } \\
\text { conhecimento }\end{array}$ & $\begin{array}{l}\text { Conhecimento } \\
\text { desenvolvido e mantido } \\
\text { em cada unidade }\end{array}$ & $\begin{array}{l}\text { Conhecimento } \\
\text { desenvolvido e } \\
\text { mantido no centro }\end{array}$ & $\begin{array}{l}\text { Conhecimento } \\
\text { desenvolvido no } \\
\text { centro e transferido } \\
\text { para unidades }\end{array}$ & $\begin{array}{l}\text { Conhecimento } \\
\text { desenvolvido em } \\
\text { conjunto e compartilhado } \\
\text { em todo o mundo }\end{array}$ \\
& & no exterior & &
\end{tabular}

Fonte: Adaptado de BARTLETT; GOSHAL (1992, p. 94).

Para os autores o modelo que melhor responde ao cenário global é o da organização transnacional. Porém, "a organização precisa ser capaz de equilibrar a diversidade de perspectivas e aptidões [...] e assegurar que nenhum grupo administrativo isolado domine os outros." Dadas as diferenças de papéis e responsabilidades das unidades organizacionais, a direção precisa criar um conjunto de processos flexíveis de coordenação para que cada unidade e tarefa sejam administradas da maneira mais apropriada, porém sem perder suas características e sua integração (BARTLETT; GHOSHAL, 1992, p. 95).

De acordo com Yip (1996, p. 178), é preciso observar que nenhuma organização conseguiu uma solução totalmente satisfatória para a questão do "pensar globalmente e agir localmente". Apesar de algumas terem conseguido criar elementos na área de gestão global com bastante sucesso. O que ocorre, como declaram Ansoff e Mcdonell (1993, p. 258), é que quando uma organização opera num ambiente doméstico estável, a sua atenção administrativa está concentrada na competição e na tecnologia que determinam o sucesso no mercado.
Quando essa organização passa a atuar fora do seu mercado nacional, seu pensamento administrativo está voltado para novos concorrentes e a uma dinâmica de competição diferente. Porém, além das variáveis de competição, alertam Ansoff e Mcdonell (1993), o sucesso em outros mercados também é determinado por outros fatores que permanecem em plano secundário, e que nem sempre são levados em conta. Alguns desses fatores são de cunho econômico, cultural e político. Do ponto de vista dos fatores culturais, que são de maior interesse neste trabalho, os autores colocam que a atuação da organização em novos ambientes pode diferirquanto ao gosto dos consumidores, capacidade dos clientes de assimilar tecnologia, nível de conhecimento tecnológico da força de trabalho, estilo de liderança e de trabalho que os profissionais praticam, valores e crenças nacionais entre outros.

$\mathrm{Na}$ sociedade atual, segundo Ansoff e Mcdonell (1993, p. 472), existem poucas organizações nas quais a estrutura de poder é "monolítica e concentrada numa única pessoa ou único grupo, com uma cultura uniforme permeando toda a organização". Em grande parte, as organizações são multiculturais e possuem 
vários centros de poder. Sendo assim, qualquer mudança ou decisão poderá provocar uma multiplicidade de reações frente às estratégias que podem ser tanto positivas quanto negativas.

Além do mais, os consumidores envolvidos neste relacionamento também são de culturas diferentes como comentam Ruben, Serva e Castro (1996, p. 78), “devido à globalização e ao mesmo tempo a manutenção de tradições locais e nacionais, a cultura torna-se uma dimensão que, se não for equacionada devida e antecipadamente, é capaz de determinar fracassos" e também podemos dizer que pode potencializar as diferenças.

\section{A cultura nacional e as organizações}

A idéia de cultura, para Santos (2003, p. 23), está associada a um dos campos do saber institucionalizados no Ocidente: as humanidades. Ela é "baseada em critérios de valor, estéticos, morais ou cognitivos que se definindo a si próprios como universais, elidem a diferença cultural". E o autor comenta que a globalização contribuiu para o aumento e aprofundamento das diferenças.

A partir da década de 80, as abordagens das ciências humanas e sociais convergiram para o campo interdisciplinar dos estudos culturais para pensar a cultura como um fenômeno associado a repertórios de sentido ou de significado partilhados pelos membros de uma sociedade, mas também associado à diferenciação e hierarquização, no quadro de sociedades nacionais, de contextos locais ou de espaços transnacionais. A cultura tornou-se, assim, um conceito estratégico central para a definição de identidades e de alteridades no mundo contemporâneo (SANTOS, 2003).

Hall (1998) defende o argumento de que as identidades nacionais não nascem com as pessoas, mas sim desenvolvidas e transformadas no interior da "representação". O país não é apenas uma entidade política, mas algo que produz significado é um "sistema de representação cultural". E que as culturas nacionais são uma forma moderna, pois a lealdade e a identificação que eram, em sociedades mais tradicionais, dadas à tribo, ao povo, à religião e a região, foram transferidas, de forma gradual, à cultura nacional.
Da mesma forma, ao se falar de organização e sua cultura é preciso analisar os pressupostos de cultura nacional que ela carrega. Os autores Motta e Caldas (1997, p. 18) comentam que um dos fatores mais importantes de diferenciação de culturas organizacionais é a cultura nacional. Para eles os "pressupostos básicos, os costumes, as crenças e os valores, bem como os artefatos que caracterizam a cultura de uma empresa, trazem sempre, de alguma forma, a marca de seus correspondentes na cultura nacional". Não há como, portanto, estudar a cultura das organizações que estão inseridas numa sociedade, sem estudar a cultura - ou as culturas - dessa sociedade.

Um trabalho muito citado por autores de gestão internacional e de organizações é de Hofstede (1984). Em sua pesquisa, sobre as diferenças culturais entre países das subsidiárias de uma corporação multinacional (IBM) em 64 países e o impacto delas na cultura organizacional, o holandês Geert Hofstede estabeleceu algumas dimensões para comparação entre culturas. A descoberta mais importante de Hofstede, que ampliou os estudos sobre o assunto, refere-se à importância da cultura nacional na explicação das diferenças de atitudes e valores em relação ao trabalho. Normalmente, outros fatores eram tidos como mais importantes, como: posição na organização, profissão, idade ou gênero.

De acordo com Ruben, Serva e Castro (1996), a organização está inserida em uma sociedade e, desta forma, está permeada pela cultura desta sociedade. Muitos teóricos já reconhecem o papel que a cultura representa nos indivíduos que fazem parte de uma organização e, em conseqüência o quanto a cultura destes indivíduos afeta o dia-adia organizacional. Além disso, cada organização tem suas características, normas e valores que constituem a sua própria cultura (FREITAS, 1991; MOT'TA; CALDAS, 1997; GHOSHAL;TANURE, 2004; BARROS, 2003).

Segundo Bartlett e Ghoshal (1992, p. 61), a influência da história, a infra-estrutura e a cultura de uma nação permeiam todos os aspectos da vida no país, incluindo as normas, valores e comportamentos dos gerentes nas organizações nacionais. As características comportamentais que são influenciadas pelo país tornam-se parte arraigada do "modo de ser" de cada organização e moldam a estrutura e os processos de sua extensão internacional. 
Em contrapartida, Freitas (1991) argumenta que vivemos atualmente numa sociedade organizacional: "estando em qualquer parte do mundo, as grandes organizações são capazes de influenciar a maior parte do dia-a-dia das pessoas". Por isso, os autores Motta e Caldas (1997, p. 16), acreditam que mesmo com o potencial de diversidade de culturas que podem ser geradas dentro das sociedades, é também verdade que, com a globalização, há tendência para profunda uniformização nas classes dominantes e médias de todo o mundo. Essa uniformização começa nas organizações onde a "ideologia tecnocrática instaurou um mundo muito semelhante de racionalidade e de comportamento".

De outro lado, toda sociedade, em maior ou menor grau, filtra e adiciona seu próprio "tempero" a idéias e tecnologias globalizantes criando suas próprias versões. Por sua vez, organizações também re-filtram e retemperam essas idéias, costumes e valores globalizados, mas já nacionalizados, a sua própria maneira.

Num primeiro momento, a impressão que se tem ao olhar a realidade organizacional brasileira é de estar presenciando fenômenos gerenciais típicos, muito parecidos com quaisquer outros observados em vários lugares do mundo. Porém, Caldas e Wood Jr. (1999, p. 29) postulam que é fundamental um olhar mais profundo dos "traços elementares da cultura nacional" para entender os fenômenos por trás da fachada. A textura sócio-cultural brasileira é complexa e multifacetada, influenciando as organizações e a forma como são geridas.

Muitos autores sobre gestão de empresas, gestão internacional e estratégia internacional incluírem a cultura nacional nos seus trabalhos. No contexto da produção brasileira, temos ainda poucos trabalhos que abordam a perspectiva da cultura nacional e sua influência na gestão e na internacionalização das organizações. Dentre os trabalhos que abordam esta perspectiva podemos destacar Freitas (1991) Motta e Caldas (1997), Ghoshal e Tanure (2004), Barros (2003), Barros e Prates (1996) e Rodrigues (1999) entre outros. Estes trabalhos são de grande relevância e nos dão pistas para compreender a realidade brasileira. Porém, ainda são poucos e há muito por se "descobrir".

\section{METODOLOGIA}

O trabalho científico necessita apoiarse em um referencial metodológico sólido. Pois, ele norteia o processo de pesquisa a partir da explicitação do fenômeno a ser explorado, passando pelos procedimentos usados na investigação até a explicação dos resultados e das conclusões obtidas.

Desta forma, a metodologia científica pode ser entendida, de acordo com Santos (2002, p. 161), como o "estudo e desenvolvimento de ferramentas facilitadoras da produção e apresentação de conhecimentos".

Este trabalho é de cunho qualitativo e como tal preocupa-se com a verificação dos múltiplos aspectos de uma dada realidade (GIL, 1999). Além disso, a pesquisa qualitativa possibilita "perceber o nível dos significados, motivos, aspirações, atitudes, crenças e valores, que se expressa pela linguagem comum e na vida cotidiana" (MINAYO; SANCHES, 1993, p. 245).

$O$ presente artigo configura-se como uma pesquisa exploratória, pois busca "informar ao pesquisador a real importância do problema, o estágio em que se encontram as informações já disponíveis a respeito do assunto", conforme Santos (2002, p. 26), e para isso utilizou-se a fonte de dados bibliográfica constituída de artigos de anais de eventos e periódicos acadêmicos. $\mathrm{O}$ critério de escolha das fontes de pesquisa baseouse na classificação dos mesmos diante do Sistema Qualis, da Capes, optando-se por aqueles classificados como Nacionais - A e que apresentassem trabalhos dentro do escopo da Gestão Internacional e Estratégia Internacional. Desta foram, buscou-se garantir um nível de qualidade dos trabalhos pesquisados.

As fontes pesquisadas foram: Revista de Administração de Empresas (RAE), RAE Eletrônica e Revista de Administração Contemporânea (RAC); os anais do Encontro da Associação Nacional dos Programas de Pós Graduação no Brasil (EnANPAD); e os anais do Encontro de Estudos de Estratégia (3 E's). A pesquisa abrangeu um período de seis anos compreendidos entre 2001 e 2006. A escolha deste período específico deu-se pelo fato de ter sido criada a partir de 2001 a área de 
Gestão Internacional no EnANPAD e, a partir daí, concentrar os trabalhos sobre o assunto.

Quanto à escolha dos artigos utilizados neste trabalho, o principal critério foi que apresentassem contribuições para o conhecimento da gestão internacional no contexto brasileiro, abordando aspectos da cultura nacional e sua influência nas organizações.

É importante salientar que este trabalho não tem a pretensão de esgotar o assunto ou assumir que os artigos aqui estudados são os únicos ou os melhores em detrimento de outros que não foram citados. Eles foram escolhidos também com base nas percepções dos autores e, portanto, sujeitos a vieses.

\section{RESULTADOS OBTIDOS}

O maior volume de artigos encontrados foi nos anais do EnANPAD. É interessante ressaltar que antes do ano de 2001, havia uma área chamada de Administração Estratégica e que a partir desse ano passa ser dividida em duas áreas distintas chamadas de Estratégia e de Gestão Internacional. Neste trabalho foram pesquisados artigos somente da área de Gestão Internacional. A Tabela 2 demonstra o total de artigos dessa área durante os anos de 2001 até 2006, a quantidade de artigos que foram escolhidos para análise em profundidade e o percentual destes artigos com relação ao total.

TABELA 2 - Artigos do ENANPAD de 2001 a 2006

\begin{tabular}{lllllll}
\hline \multicolumn{7}{c}{ Anais do ENANPAD - Área: Gestão Internacional } \\
\hline ANO & 2001 & 2002 & 2003 & 2004 & 2005 & 2006 \\
Total de artigos & 10 & 17 & 22 & 26 & 26 & 28 \\
Selecionados & 2 & 7 & 3 & 6 & 3 & 4 \\
$\%$ do total & $20 \%$ & $41 \%$ & $13 \%$ & $23 \%$ & $11 \%$ & $14 \%$ \\
\hline
\end{tabular}

Fonte: Os autores.

Como pode ser observada na tabela acima, a área de Gestão Internacional apresentou crescimento ao longo dos anos no número total de artigos. Porém, tal crescimento não ocorreu no número de artigos que abordam a perspectiva da cultura e sua influência no ambiente organizacional.
A pesquisa nos anais do 3 E's compreendeu apenas dois anos, visto que é um evento bianual. Os artigos pesquisados foram dos anos 2003 e 2005. A Tabela 3 apresenta o total de artigos de cada ano, bem como o número de artigos escolhidos e o percentual destes com relação ao total de artigos de cada ano.

TABELA 3 - Artigos do E's de 2003 e 2005

\begin{tabular}{lll}
\hline & Resultado da Pesquisa nos Anais do 3 E's \\
\hline ANO & 2003 & 2005 \\
Total artigos & 96 & 100 \\
Selecionados & 1 & 5 \\
PERC. DO TOTAL $(\%)$ & $1 \%$ & $5 \%$ \\
\hline
\end{tabular}

Fonte: Os autores.

Percebe-se que houve um aumento de um ano para o outro, configurando-se em 5 vezes mais artigos que consideram o contexto cultural nacional e organizacional, porém, o número ainda é tão pequeno que no âmbito geral mostra-se como pouco significativo diante do total de artigos nas duas edições do evento. 
A pesquisa realizada nas revistas RAC, RAE e RAE Eletrônica também encontrou panorama semelhante ao encontrado nos anais dos eventos. $\mathrm{Na}$ RAC foram encontrados sete artigos, sendo um em 2001; três em 2002; um em 2003 e dois em 2005. Na RAE foi encontrado apenas um artigo, de 2005. E na RAE Eletrônica não foi encontrado nenhum artigo que destacasse a cultura brasileira.

Estas revistas abordam temas das diversas áreas da Administração, além de terem um natural viés por determinados assuntos, sendo que o tema gestão internacional é só um deles. Destarte, é coerente o fato de apresentarem um número reduzido de artigos sobre o assunto e ainda mais reduzido seja o número de artigos que abordem a gestão internacional no contexto da cultura brasileira.

Os números, de maneira geral, demonstram que a cultura nacional brasileira é, ainda, pouco explorada. A superioridade numérica dos artigos que tratam o assunto mais de forma prescritiva leva a percepção de que o pensamento funcionalista predomina, no sentido de testar e verificar modelos e teorias no âmbito organizacional com maior preocupação com o resultado do modelo per si.

Foram encontrados dois trabalhos cujo objetivo era traçar um panorama dos estudos sobre estratégia no Brasil, coincidentemente, os dois trabalhos são do ano de 2001 e utilizam a mesma base de dados: os 127 artigos do EnANPAD do período de 1997 ao ano 2000. O período de estudo dos dois trabalhos não contempla a divisão de Gestão Internacional dentro da área de estratégia. Mesmo assim, eles ajudam a entender o porquê do pequeno número de trabalhos que estudam a perspectiva cultural, tanto no âmbito nacional quanto organizacional.

No primeiro trabalho os autores Paulino et al. (2001) analisaram alguns traços característicos do "pensar" estrategicamente no Brasil e comentam que é comum os empresários de pequenas e médias empresas nacionais e consultores buscarem constantemente modernas técnicas gerenciais como possibilidade de solução às pressões da concorrência ou tomadas como um "milagroso" caminho de modernização da estrutura organizacional sem, contudo, preocupar-se com a adaptação à realidade brasileira, seu estilo de gerenciar e a influência da cultura nacional. Os autores argumentam, ainda, que o cenário econômico/industrial brasileiro sofre as influências econômicas e tecnológicas dos grandes centros industriais americano e europeu onde predomina a visão de "alta competitividade para um cenário globalizado". Em decorrência desse fato pode-se concluir que esse cenário seja um modelo de inspiração aos acadêmicos brasileiros que acabam adotando teorias estratégicas que priorizam a questão da "competitividade" como os modelos conceituais de origem americana.

Neste estudo, os autores analisaram a produção acadêmica sobre o tema sob a luz das escolas de estratégia de Mintzberg, Ahlstrand e Lampel (2000). A análise dos autores mostra que dentre os 127 trabalhos analisados, 25,2\% apresentaram um enfoque predominante na escola de Posicionamento, o que sinaliza que a tipologia de Porter é um importante referencial de estudo. Este dado também reflete a predominância do aspecto competitivo nos estudos estratégicos que se concentram em modelos prescritivos. Aparecem em segunda e terceira posição, respectivamente com $14,2 \%$ e $13,4 \%$ do interesse de estudos apresentados no período, as escolas Ambiental e de Poder que propõem como um dos fatores de análise o referencial externo. Do trabalho resulta a percepção quanto ao jeito brasileiro de administrar que é marcado por enorme heterogeneidade no cenário nacional, convivem grandes organizações ao lado de outras, muito aquém do que se pode considerar "excelência gerencial".

O outro trabalho, de Bignetti e Paiva (2001), analisa a produção brasileira sobre estratégia por meio das linhas de pensamento predominantes: "dentro da visão determinista, o ambiente é descrito como sendo completamente distinto e separado da organização, existindo claras fronteiras delimitando a ambos. $\mathrm{Na}$ visão indeterminista, coloca-se a idéia de que a organização e o ambiente não são completamente separados e independentes, mas que pertencem ao mesmo continuum". Como resultado, os autores apontam para uma "predominância de uma visão determinista do ambiente, em que Porter é um dos autores mais citados. A visão indeterminista do ambiente aparece como uma perspectiva que ainda atrai poucos pesquisadores".

Outra observação importante foi a de que autores nacionais são pouco citados, o que demonstra uma limitação da pesquisa acadêmica brasileira desta área e leva a algumas considerações 
sobre as possíveis causas como a "posição colonizadora" dos pesquisadores brasileiros, além do número reduzido de pesquisadores, aliado a sua falta de integração (BIGNETTI; PAIVA, 2001, p. 10). Estes mesmos autores publicaram um artigo na Revista de Administração Contemporânea - RAC - ampliando em um ano esta pesquisa, perfazendo um total de 185 artigos pesquisados entre 1997 e 2001 e chegam a conclusões semelhantes ao trabalho anterior (BIGNETTI; PAIVA, 2002).

Apesar de quantitativamente não ser tão expressiva, a produção acadêmica brasileira que retrata a gestão internacional no contexto da sua cultura nacional apresenta pontos de contato interessantes e considerações que contribuem para o entendimento do que é a nossa realidade nessa área específica. Sendo assim, foram apontados aqui os elementos destacados dentre o material pesquisado que contribuem para o entendimento da gestão internacional sob a ótica da cultura nacional no ambiente organizacional.

É possível salientar alguns tópicos abordados como: a cultura nacional e organizacional relacionadas com a competitividade das organizações; as dimensões culturais e a estratégia; a internacionalização de empresas; a expatriação e o ajuste intercultural; estratégias internacionais de marketing, de recursos humanos e de negociação; fusões e aquisições; e práticas organizacionais.

Sobre o tema da cultura e a competitividade, o artigo de Mello e Cunha (2001) aborda a vantagem competitiva e as organizações brasileiras. A proposta dos autores é criar uma agenda de pesquisa no contexto nacional para entender as organizações sobre a perspectiva das capacidades organizacionais. Elas são uma extensão da perspectiva da competição baseada em recursos. Os autores justificam esta necessidade com o argumento de que além das peculiaridades econômicas, ressaltam-se as características culturais que moldam o comportamento dos indivíduos e que são específicas do contexto brasileiro. Além do que, uma agenda de pesquisa desenvolvida em simetria com a produção acadêmica internacional facilitaria o diálogo.

Já o trabalho de Queiroz, Álvares e Moreira (2005) aborda a diversidade cultural e a necessidade de melhor compreendê-la no âmbito organizacional. Os autores argumentam que o sucesso da gestão da diversidade deve-se a fatores contingenciais como a estratégia e a cultura. Como resultado de uma pesquisa feita em uma multinacional do setor automobilístico, os autores concluem com a afirmação de que "em determinados contextos podese esperar que uma adequada gestão da diversidade cultural venha a representar um recurso estratégico capaz de fazer aumentar a performance das empresas". Neste caso, também pode ser citado o trabalho de Pardini (2005) que trata da formação cultural como construto de ligação entre a visão empreendedora e a ação estratégica.

Outro trabalho é o de Jacometti e Machado-da-Silva (2005) que relacionam a cultura organizacional e dependências de poder na definição de estratégias. Os resultados obtidos demonstram que as crenças e os valores de maior significado influenciam as estratégias de maior importância. Também ressaltam que as diferenças no que concerne à atribuição de importância são dadas pelos grupos às crenças e valores e explicam os diversos posicionamentos frente as mesmas pressões ambientais.

Foram identificados alguns trabalhos que contribuem com o debate sobre as dimensões culturais nas organizações e que tratam tanto da cultura no seu aspecto nacional quanto organizacional. O artigo de Hilal (2003) tem o propósito de identificar as dimensões culturais segundo Hofstede, as idiossincrasias e a força da cultura organizacional de uma empresa brasileira com atuação internacional. Os resultados apontam para a influência da cultura nacional na cultura organizacional, as dimensões encontradas refletem a ambigüidade e o tênue limite ético característicos da cultura brasileira. Já no estudo de Silva (2002), também com base no trabalho de Hofstede, e que procurou identificar as dimensões culturais predominantes em empresas que receberam o Prêmio Nacional de Qualidade - PNQ. A análise dos dados de sete empresas apresentou os seguintes resultados: Distância do Poder e Aversão à Incerteza com grau baixo; a dimensão Coletivismo ao invés de Individualismo; a Dimensão de Feminilidade ao invés de Masculinidade; e Orientação de Tempo para o longo prazo. Com isso a autora pode concluir que estas dimensões culturais facilitam a adoção de modelos de gestão da qualidade, pois facilitam a sua obtenção e a manutenção da melhoria contínua. 
Outros trabalhos interessantes que também tratam de dimensões culturais podem ser citados como o de Boehe e Balestero (2002), sobre os valores culturais e os custos de transação; o trabalho de Blundi e Rocha (2001) sobre a diferença cultural, etnocentrismo e alteridade; e o de Spink (2002) sobre o lugar do lugar na análise organizacional.

Um dos temas de maior destaque no número de artigos é sobre a internacionalização de empresas brasileiras. Os autores Meyer Jr., Meyer e Murphy (2006) falam sobre a influência da cultura nos negócios internacionais, indicando que a leitura do contexto cultural e a busca pelo ajuste a este contexto evitam problemas que podem afetar a performance das organizações.

O trabalho de Francisco (2004) retrata as proximidades da cultura brasileira e angolana destacando as dificuldades de gestão de uma empresa brasileira e o maior compromisso para com seus funcionários que são gerados não pelas diferenças culturais, mas pelo fato do país estar em guerra. Por outro lado, Dal Bello e Gomes (2002) apresentam um estudo de caso que demonstra que apesar da existência dos fatores impeditivos que são os aspectos conjunturais, estruturais e históricos do processo de internacionalização de empresas nacionais, "há aquelas que rompem com essas barreiras e conseguem, com sucesso, a internacionalização."

No trabalho de Freitas, Blundi e Casotti (2002) são discutidas "as características do processo de internacionalização das empresas brasileiras a partir do caso de uma churrascaria, a Plataforma." Um aspecto identificável neste trabalho, e que diz respeito a cultura brasileira, é a intimidade entre funcionários e clientes, a descontração, o clima de "estamos em família", ou seja, a tendência dos brasileiros de personalizar as relações que, "se levada em consideração pelos empresários, pode vir a se tornar importantes diferenciais competitivos no mercado externo."

O trabalho de Veiga e Rocha (2001) trata da escolha de mercados, distância cultural e comprometimento em organizações brasileiras em processo de internacionalização. Os autores investigaram o processo de internacionalização de grandes empresas brasileiras a partir do modelo comportamental de internacionalização inicialmente proposto por Johanson e Wiedersheim-Paul e Johanson e Vahlne, da chamada escola nórdica.
Foram realizados três estudos de caso e investigadas questões que cobriam os aspectos sobre o início do processo de internacionalização; quais as formas adotadas para ingresso em mercados externos; qual a relação entre a seqüência da escolha dos mercados com o conceito de distância cultural; como evoluiu o comprometimento com os mercados externos; e como ocorreu o desenvolvimento de estruturas internas específicas e complexas para gerenciar o processo de internacionalização. Uma das conclusões foi que as teorias comportamentais de internacionalização dos autores da escola nórdica "não explicam adequadamente o processo de internacionalização das três empresas brasileiras estudadas. Embora alguns aspectos eventualmente se ajustem aos pressupostos e padrões indicados pela teoria, isso ocorre apenas de forma tênue e esporádica." Outra conclusão importante é sobre a "evolução das estruturas e sobre o papel das networks pessoais na internacionalização de empresas brasileiras."

Como contraponto do trabalho de Veiga e Rocha (2001), os autores Hilal e Hemais (2003) apresentam um artigo que trata justamente da internacionalização na ótica da escola nórdica e empresas brasileiras. Embasados no conjunto teórico criado pela Escola Nórdica de Negócios Internacionais, os autores desenvolveram pesquisas em algumas empresas brasileiras, "a procura de pontos de convergência entre os modos suecos e brasileiros de expansão das firmas além fronteiras". Concluíram, através das evidências empíricas, que "se confirmam os principais pontos advogados pela escola". Porém, a diferença nos resultados obtidos nos dois artigos corrobora a necessidade de ampliar as pesquisas no Brasil sobre esta temática e o quão importantes e influentes podem ser as diferenças nacionais e regionais.

O modelo da escola nórdica de internacionalização demonstra a questão dos pontos de contato entre os temas e a necessidade de estudos mais aprofundados.

Outra temática que se destaca diz respeito sobre executivos e funcionários expatriados no Brasil e a necessidade de ajuste intercultural ou mesmo à nova cultura. Nesse sentido, os artigos selecionados contribuem para a compreensão do ambiente organizacional brasileiro, os traços culturais presentes, os choques e conflitos decorrentes das diferenças culturais. Os trabalhos 
de Homem (2005) e Homem e Tolfo (2004) tratam do ajuste cultural de expatriados no Brasil. A seleção, o treinamento e a identificação de profissionais com perfil adequado para uma expatriação precisam ser eficazes, pois envolvem altos investimentos financeiros e psicológicos. Assim, foram identificados os fatores relacionados ao ambiente estrangeiro que contribuem ou limitam o ajuste intercultural de expatriados como o idioma, o sistema político e econômico no país anfitrião.Os pontos de contato podem estar justamente na cultura que irá receber a organização, sendo ela também, um elemento facilitador do processo.

No seu artigo, Bueno, Domingues e Del Corso (2004) apresentam uma pesquisa sobre o processo de expatriação em uma multinacional do ramo automobilístico e como este processo auxilia a integração cultural. Os resultados obtidos revelaram que o processo de expatriação da subsidiária brasileira não tem atuado como instrumento de integração de culturas, pois apenas atua junto aos funcionários nos aspectos de infra-estrutura e logística de acomodação, e de forma superficial na preparação dos indivíduos para a convivência e adaptação ao contexto brasileiro. Por outro lado, foram percebidas características da cultura brasileira que facilitaram o ajuste de estrangeiros como a simpatia, a flexibilidade e a paciência.

Também podem ser destacados os trabalhos de Gonçalves e Miura (2004) sobre as implicações da cultura brasileira na expatriação de executivos; o trabalho de Dupuis e Davel (2002) que tratou da administração intercultural e a ambigüidade e adaptabilidade como veículos sociais; Santos (2002) e os aspectos da expatriação de brasileiras nos Estados Unidos; bem como, Rodriguez (2002) sobre grupos multiculturais e interação e o trabalho de De Paula e Staub (2006) sobre a percepção de executivos italianos quanto a gestão brasileira.

$O$ aspecto de internacionalização de empresas também aparece relacionado às estratégias de marketing, de negociação e de recursos humanos. Corroborado pelo trabalho de Giraldi, Maschieto e Carvalho (2005) que trata da competitividade mercadológica internacional e a imagem do Brasil. O trabalho de Motta (2004) destaca-se por abordar o ambiente cultural como modelador das decisões de marketing. No seu artigo, Floriani (2004) relaciona os valores de uma organização e suas formas de negociação internacional. Crnkovic (2003) apresenta um trabalho onde a cultura organizacional é colocada como diferencial estratégico em recursos humanos.

Um tema também mencionado é sobre fusões e aquisições e os impactos da cultura local. Destacam-se trabalhos das autoras Tanure e Cançado (2005a) que, por meio de uma pesquisa realizada com 106 empresas e 7 estudos de caso, argumentam que os dados sugerem que existe um modelo híbrido de gestão que é influenciado pela cultura brasileira onde são combinados os processos racionalizados das adquirentes com a informalidade e as relações personalizadas adquiridas. As autoras também apontam que é preciso ter cuidado com as etapas do processo, respeitando a cultura local.

Em trabalho semelhante, as autoras apresentam resultados do impacto da cultura local no processo de aquisição de dois casos, mostrando o continuum que se forma: em um extremo um maior grau de profissionalização das adquirentes e de outro uma gestão personalizada das empresas adquiridas (TANURE; CANÇADO, 2005b). Outros trabalhos que podem ser citados são o de Rossini; Crubellate e Mendes (2001) sobre a reação cultural à aquisição e as suas conseqüências para o processo; o artigo de Sayo, Tanure e Duarte (2006) sobre as mudanças na cultura pós-aquisição; e o trabalho de Oltramari, Homem e Bispo (2006) sobre a mudança na gestão de uma empresa familiar adquirida por uma multinacional e o papel da área de gestão de pessoas no processo.

Alguns trabalhos relacionam a cultura nacional ao desempenho e as práticas organizacionais. São exemplos os trabalhos de Musiello Neto (2002) sobre cultura organizacional e desempenho. O trabalho de Duarte (2002) relaciona a cultura nacional com as práticas organizacionais. Também se destaca o trabalho de Brito e Vasconcelos (2005) sobre o desempenho das empresas brasileiras e a influência do país de origem.

Com o intuito de sintetizar os principais temas abordados e seus respectivos autores, foi criada a Tabela 4. Para não caracterizar nenhuma escala de importância dos temas e autores, foi utilizada a classificação alfabética de temas. 
TABELA 4 - Relação dos tópicos abordados e seus autores

\begin{tabular}{ll}
\hline Tópicos Abordados & Autores Relacionados \\
\hline $\begin{array}{l}\text { Competitividade } \\
\text { Cultura e Poder }\end{array}$ & Mello e Cunha (2001); \\
Dimensões Culturais e Estratégia & Jacometti e Machado-da-Silva (2003); \\
& Hilal (2003); Silva (2002); Boehe e Balestero (2002); \\
& Blundi e Rocha (2001); Spink (2002) \\
Diversidade Cultural & Álvares e Moreira (2005); Pardini (2005) \\
Expatriação e Ajuste Intercultural & Homem (2005); Homem e Tolfo (2004); Bueno; Domingues e \\
& Del Corso (2004); Gonçalves e Miura (2004); Dupuis e Davel (2002); \\
& Santos (2003); Rodriguez (2002); De Paula e Staub (2006) \\
Estratégias de Mkt, RH e Negociação & Giraldi, Maschieto e Carvalho (2005); Motta (2004); Floriani (2004); \\
& Crnkovic (2003) \\
Fusões e Aquisições & Tanure e Cançado (2005a); Tanure e Cançado, (2005b); Rossini; \\
& Crubellate e Mendes (2001); Sayão; Tanure e Duarte (2006); \\
& Oltramari; Homem e Bispo (2006) \\
& Meyer Jr, Meyer e Murphy (2006); Francisco (2004); Dal Bello e \\
Gomes (2002); Freitas; Blundi e Casotti (2002); Veiga e Rocha
\end{tabular}

Fonte: Os autores.

\section{CONCLUSÃO}

Como resultante da busca empreendida nos anais do EnANPAD, do $3 \mathrm{E}^{\text {'s }}$ e nas revistas RAC, RAE e RAE Eletrônica, em um período de seis anos compreendidos entre 2001 e 2006, pode-se ressaltar pontos considerados pertinentes para o objetivo deste artigo como: o baixo número de artigos que retratam a realidade da cultura; a maioria dos artigos relata o sucesso ou o insucesso de teorias, abordagens e modelos de estratégias, sem demonstrar muita preocupação com a cultura nacional ou a necessidade de adequação para os padrões brasileiros. Aqueles artigos que trazem a tona à questão do contexto brasileiro enquadram-se em tópicos específicos, sendo que vários deles estão relacionados a vários temas dentro da Gestão Internacional.

Com relação ao primeiro e ao segundo pontos, é possível perceber, uma correlação com as conclusões de Bignetti e Paiva (2001, 2002). Estes autores fizeram levantamentos sobre a produção brasileira de estratégia, em um período diferente do pesquisado neste trabalho, onde classificam a postura do pesquisador brasileiro como de "colonizado", ou seja, preocupado em reproduzir e aplicar teorias e práticas importadas de países desenvolvidos tidos como exemplo de eficácia e de sucesso como é o caso dos modelos norte-americanos que têm um caráter mais prescritivo e que pouco levam em conta as questões de diferenças culturais.

Uma possível causa do baixo número de artigos com a perspectiva da cultura nacional e sua influência no ambiente organizacional pode ser a dificuldade dos executivos e, de uma parte da academia, em trabalhar a subjetividade. A necessidade de mostrar resultados tangíveis no menor tempo possível e a falsa impressão de homogeneização das ferramentas e práticas de gestão fazem com que sejam deixados de lado os aspectos culturais e sua influência na formulação, implementação e resultados da gestão internacional.

Daqueles artigos selecionados, por abordarem a questão cultural, apesar das diferenças epistemológicas que possam existir entre as fontes pesquisadas, destacam-se dentre suas principais temáticas: a competitividade e 
desempenho das organizações; as dimensões culturais e a estratégia; a internacionalização de empresas; a expatriação e o ajuste intercultural; estratégias internacionais de marketing, de recursos humanos e de negociação e; fusões e aquisições. É possível perceber que alguns temas têm um grau de maturidade maior do que outros, possibilitando a continuidade de pesquisas dentro da mesma linha e análise comparativa de dados.

Algumas contribuições são bastante pertinentes como a proposta de criar uma agenda de pesquisa no contexto nacional para entender as organizações sobre a perspectiva das capacidades organizacionais, pois além das peculiaridades econômicas, as características culturais moldam o comportamento dos indivíduos e são específicas do contexto brasileiro. Além do que, uma agenda de pesquisa desenvolvida em simetria com a produção acadêmica internacional facilitaria o diálogo.

Em se tratando de experiências de estrangeiros no Brasil, foram reportadas características da cultura brasileira que facilitaram o ajuste de estrangeiros como a simpatia, a flexibilidade e a paciência. Outros resultados reforçam o argumento de que alguns traços culturais presentes no nosso cotidiano também aparecem no ambiente organizacional como a ambigüidade e a facilidade na adoção de modelos e práticas de gestão. Também foram percebidos traços como a descontração, o clima de camaradagem, a tendência em personalizar as relações de trabalho, além da facilidade de adaptação. Aspectos estes que podem ser considerados pontos de contato quando o tema é Gestão Internacional e cultura nacional brasileira.

Alguns trabalhos apontam para a complexidade da cultura brasileira que não é facilmente decifrada por modelos pré-estabelecidos e simplificadores. Igualmente importante é a percepção das enormes diferenças culturais entre as regiões brasileiras.

\section{REFERÊNCIAS}

ANSOFF, H. I.; MCDONNELL, E. J. Implantando a administração estratégica. 2. ed. São Paulo: Atlas, 1993.

BARROS, B. T. Gestão à brasileira: uma comparação entre América Latina, Estados Unidos, Europa e Ásia. São Paulo: Atlas, 2003.
BARROS, B. T; PRATES, M. A. S. O estilo brasileiro de administrar. São Paulo: Atlas, 1996.

BARTLETT, C. A.; GHOSHAL, S. Gerenciando empresas no exterior: a solução transnacional. São Paulo: Makron Books, 1992.

BIGNETTI, L. P.; PAIVA, E. L. XXV Estudo das citações de autores de estratégia na produção acadêmica brasileira. In: ENCONTRO ANUAL DA ASSOCIAÇÃO NACIONAL DE PÓS-GRADUAÇÃO E PESQUISA EM ADMINISTRAÇÃO, 25., 2001, Campinas, Anais... Campinas: EnANPAD, 2001. p. 1-16.

Ora (direis) ouvir estrelas!: estudo das citações de autores de estratégia na produção acadêmica brasileira. Revista de Administração Contemporânea, v. 6. n. 1, p. 105-126, jan./abr. 2002.

BLUNDI, M. D.; ROCHA, E. Franquia, etnocentrismo e alteridade: um estudo sobre diferença cultural. In: ENCONTRO ANUAL DA ASSOCIAÇÃO NACIONAL DE PÓSGRADUAÇÃO E PESQUISA EM ADMINISTRAÇÃO, 25., 2001, Campinas. Anais... Campinas, 2001. p. 1-16.

BOEHE, D. M.; BALESTERO, M. V. Um estudo comparativo dos valores culturais e suas implicações para os custos de transação. In: ENCONTRO ANUAL DA ASSOCIAÇÃO NACIONAL DE PÓS-GRADUAÇÃO E PESQUISA EM ADMINISTRAÇÃO, 26., 2002, Salvador. Anais... Salvador: EnANPAD, 2002. p. 1-16.

BRITO, L. A. L.; VASCONCELOS, F. C. A influência do país de origem no desempenho das empresas. Revista de Administração Contemporânea, v. 9, n. 4, p. 97-118, out./ dez. 2005.

BUENO, J. M.; DOMINGUES, C. R.; DEL CORSO, J. M. O processo de expatriação como instrumento de integração de culturas em uma organização no Brasil - o caso Renault. In: ENCONTRO ANUAL DA ASSOCIAÇÃO NACIONAL DE PÓS-GRADUAÇÃO E PESQUISA EM ADMINISTRAÇÃO, 28., 2004, Curitiba. Anais... Curitiba: EnANPAD, 2004. p. 1-16. 
CALDAS, M.; WOOD Jr., T. Transformação e realidade organizacional: uma perspectiva brasileira. São Paulo: Atlas, 1999.

CASTELLS, M. A era da informação: economia, sociedade e cultura. fim de milênio. 2. ed. São Paulo: Paz e Terra, 2000. v. 3.

CRNKOVIC, L. H. Cultura organizacional: o diferencial estratégico da área de recursos humanos. In: ENCONTRO ANUAL DA ASSOCIAÇÃO NACIONAL DE PÓS-GRADUAÇÃO E PESQUISA EM ADMINISTRAÇÃO, 27., 2003, Atibaia. Anais... Atibaia: EnANPAD, 2003. p. 1-16.

DAL BELLO, U. B.; GOMES, M. M. A empresa brasileira e o desafio à internacionalização. In: ENCONTRO ANUAL DA ASSOCIACCÃO NACIONAL DE PÓS-GRADUAC ÃO E PESQUISA EM ADMINISTRAÇÃO, 26., 2002, Salvador. Anais... Salvador: EnANPAD, 2002. p. 1-16.

DE PAULA, E. R.; STAUB, I. D. A gestão brasileira revisitada por executivos expatriados italianos. In: ENCONTRO ANUAL DA ASSOCIACC̃̃O NACIONAL DE PÓSGRADUAÇÃO E PESQUISA EM ADMINISTRAÇÃO, 30., 2006, Salvador. Anais... Salvador: EnANPAD, 2006. p. 1-16.

DUARTE, R. G. The debate about national culture and organizational practices: a historical account. In: ENCONTRO ANUAL DA ASSOCIAÇÃO NACIONAL DE PÓSGRADUAÇÃO E PESQUISA EM ADMINISTRAÇÃO, 26., 2002, Salvador. Anais... Salvador: EnANPAD, 2002. p. 1-16.

DUPUIS, J. P.; DAVEL, E. Administração intercultural no novo mundo: ambigüidade e adaptabilidade como veículos sociais no Brasil e no Quebec. In: ENCONTRO ANUAL DA ASSOCIACÃO NACIONAL DE PÓSGRADUAÇÃO E PESQUISA EM ADMINISTRAÇÃO, 26., 2002, Salvador, Anais... Salvador: EnANPAD, 2002. p. 1-16.
FLORIANI, D. E. A análise dos valores sob a ótica dos negócios internacionais: um estudo ítalo-brasileiro. In: ENCONTRO ANUAL DA ASSOCIAÇÃO NACIONAL DE PÓSGRADUAÇÃO E PESQUISA EM ADMINISTRAÇÃO, 28., 2004, Curitiba. Anais... Curitiba: EnANPAD, 2004. p. 1-16.

FRANCISCO, J. M. S. D. Internacionalização para um mercado culturalmente próximo, mas em guerra: a Odebrecht em Angola. In: ENCONTRO ANUAL DA ASSOCIAÇÃO NACIONAL DE PÓS-GRADUAÇÃO E PESQUISA EM ADMINISTRAÇÃO, 28., 2004. Curitiba. Anais... Curitiba: EnANPAD, 2004. p. 1-16.

FREITAS, M. E. de. Cultura organizacional: formação, tipologias e impactos. São Paulo: Makron; McGraw-Hill, 1991.

FREITAS, H. G.; BLUNDI, M. D. S.; CASOTTI, L. M. Internacionalização da churrascaria Plataforma: peculiaridades da cultura brasileira como um diferencial competitivo. In: ENCONTRO ANUAL DA ASSOCIAÇÃO NACIONAL DE PÓS-GRADUAÇÃO E PESQUISA EM ADMINISTRAÇÃO, 26., 2002, Salvador. Anais... Salvador: EnANPAD, 2002. p. 1-16.

GHOSHAL, S.; TANURE, B. Estratégia e gestão empresarial: construindo empresas brasileiras de sucesso - estudos de caso. Rio de Janeiro: Elsevier, 2004.

GIL, A. C. Métodos e técnicas de pesquisa social. 5. ed. São Paulo: Atlas, 1999.

GIRALDI, J. M. E.; MASCHIETO, A. J.; CARVALHO, D. T. Competitividade mercadológica nos mercados internacionais: uma investigação empírica sobre a avaliação da imagem do Brasil. In: ENCONTRO DE ESTUDOS DE ESTRATÉGIA, 2., 2005, Rio de Janeiro. Anais... Rio de Janeiro: EnANPAD, 2005. p. 1-16. 
GONÇALVES, G. A.; MIURA, I. K. Executivo expatriado no Brasil: as implicações das características culturais brasileiras na adaptação no trabalho e na vida social. In: ENCONTRO ANUAL DA ASSOCIAÇÃO NACIONAL DE PÓSGRADUAÇ̃̃O E PESQUISA EM ADMINISTRAÇÃ̆O, 23., 2004. Curitiba. Anais... Curitiba: EnANPAD, 2004. p. 1-16.

HALL, S. A identidade cultural na pósmodernidade. 2. ed. Rio de Janeiro: DP\&A, 1998.

HILAL, A. Organizational culture dimensions: a Brazilian case. In: ENCONTRO ANUAL DA ASSOCIAÇÃO NACIONAL DE PÓSGRADUAÇÃO E PESQUISA EM ADMINISTRAÇÃO, 27., 2003, Atibaia. Anais... Atibaia: EnANPAD, 2003. p. 1-16.

HILAL, A.; HEMAIS, C. A. O processo de internacionalização na ótica da escola nórdica: evidências empíricas em empresas brasileiras.

Revista de Administração Contemporânea, v. 7, n. 1, p. 109-124, jan./mar. 2003.

HOFSTEDE, G. Culture's consequences: international differences in work-related values. London: Sage Publications, 1984.

HOMEM, I. D. O ajustamento intercultural de expatriados: um estudo de caso em uma multinacional brasileira do estado de Santa Catarina. In: ENCONTRO ANUAL DA ASSOCIAÇÃO NACIONAL DE PÓSGRADUAÇÃO E PESQUISA EM ADMINISTRAÇÃO, 19., 2005, Brasília. Anais... Brasília: EnANPAD, 2005. p. 1-16.

HOMEM, I. D.; TOLFO, S. R. Gestão intercultural: perspectivas para o ajustamento de executivos expatriados. In: ENCONTRO ANUAL DA ASSOCIAÇÃO NACIONAL DE PÓS-GRADUAÇÃO E PESQUISA EM ADMINISTRAÇÃO, 28., 2004, Curitiba. Anais... Curitiba: EnANPAD, 2004. p. 1-16.
JACOMETTI, M.; MACHADO-DA-SILVA, C. L. Cultura organizacional e dependências de poder na definição de estratégias: o caso da unidade de Curitiba do CEFET-PR. In: ENCONTRO DE ESTUDOS DE ESTRATÉGIA, 2., 2003, Curitiba. Anais... Curitiba: EnANPAD, 2003. p. 1-16.

MELLO, R. B.; CUNHA, C. J. C. A. A natureza e a dinâmica das capacidades organizacionais no contexto brasileiro: uma agenda para pesquisas sobre a vantagem competitiva das empresas brasileiras. In: ENCONTRO NACIONAL DA ASSOCIAÇÃO NACIONAL DOS PROGRAMAS DE PÓSGRADUAÇÃO, 25., 2001, Campinas. Anais... Campinas: EnANPAD, 2001. p. 1-16.

MEYER Jr., V.; MEYER, B. MURPHY, J. P. The influence of culture in international business. In: ENCONTRO ANUAL DA ASSOCIAÇÃO NACIONAL DE PÓSGRADUAÇÃO E PESQUISA EM ADMINISTRAÇÃO, 30., 2006, Salvador. Anais... Salvador: EnANPAD, 2006. p. 1-16.

MINAYO, M. C. de S.; SANCHES, O. Quantitativo-qualitativo: oposição ou complementaridade? Caderno de Saúde Pública, v. 9, n. 3. p. 239-262, jul./set. 1993.

MINTZBERG, H.; AHLSTRAND, B.; LAMPEL, J. Safári de estratégia: um roteiro pela selva do planejamento estratégico. Porto Alegre: Bookman, 2000.

MOTTA, S. L. S. Ambiente cultural como elemento modelador das decisões de marketing global. In: ENCONTRO ANUAL DA ASSOCIAÇÃO NACIONAL DE PÓSGRADUAÇÃO E PESQUISA EM ADMINISTRAÇÃO, 23., 2004, Curitiba. Anais... Curitiba: EnANPAD, 2004. p. 1-16.

MOTTA, F. C. P.; CALDAS, M. P. (Org.). Cultura organizacional e cultura brasileira. São Paulo: Atlas, 1997. 
MUSIELLO NETO, F. E. A relação da cultura organizacional com a performance no setor comercial do Rio Grande do Norte. In: ENCONTRO ANUAL DA ASSOCIAÇÃO NACIONAL DE PÓS-GRADUAÇÃO E PESQUISA EM ADMINISTRAÇÃO, 26., 2002, Salvador. Anais... Salvador: EnANPAD, 2002. p. $1-16$.

OLTRAMARI, A. P.; HOMEM, I. D.; BISPO, M. B. Gestão de pessoas em um processo de aquisição: ferramentas de sedução. In: ENCONTRO ANUAL DA ASSOCIAC $\tilde{O}$ NACIONAL DE PÓS-GRADUAÇÃO E PESQUISA EM ADMINISTRAÇÃO, 30., 2006, Salvador. Anais... Salvador: EnANPAD, 2006. p. 1-16.

ORTIZ, R. Mundialização e cultura. 2. ed. São Paulo: Brasiliense, 2003.

PARDINI, D. J. A formação cultural como construto de ligação entre visão empreendedora e ação estratégica - uma análise do caso Usiminas. In: ENCONTRO DE ESTUDOS DE ESTRATÉGIA, 2., 2005, Rio de Janeiro. Anais... Rio de Janeiro: EnANPAD, 2005. p. 1-16.

PAULINO, A. D. et al. Organização e estratégia: tendências de estudos no cenário nacional. In: ENCONTRO ANUAL DA ASSOCIAÇÃO NACIONAL DE PÓS-GRADUAÇÃO E PESQUISA EM ADMINISTRAÇ ÃO, 25., 2001, Campinas. Anais... Campinas: EnANPAD, 2001. p. 1-16.

QUEIROZ, A. C. S.; ÁlVARES, I. M.; MOREIRA, D. A. Gestão da diversidade cultural, estratégia e vantagem competitiva: um estudo de caso no Brasil. In: ENCONTRO DE ESTUDOS DE ESTRATÉGIA, 2., 2005, Rio de Janeiro. Anais... Rio de Janeiro: EnANPAD, 2005. p. 1-16.

ROSSINI, A. J.; CRUBELlATE, J. M.; MENDES, A. A. Reação cultural à aquisição: estudo do caso Santader/Noroeste. Revista de Administração Contemporânea, v. 5, n. 1, p. 145-164, jan./abr. 2001.

RODRIGUES, S. Competitividade, alianças estratégicas e gerência internacional. São Paulo: Atlas, 1999.
RODRIGUEZ, C. L. Interactions in small groups: an investigation of joint organizational sensemaking by brazilians and americans. In: ENCONTRO ANUAL DA ASSOCIAÇÃO NACIONAL DE PÓS-GRADUAÇÃO E PESQUISA EM ADMINISTRAÇÃO, 26., 2002, Salvador. Anais... Salvador: EnANPAD, 2002. p. 1-16.

RUBEN, G.; SERVA, M.; CASTRO, M. L. Resíduos e complementaridade: das relações entre a teoria da administração e a antropologia. Revista de Administração Pública, v. 30, n. 3, p. 68-80, maio/jun. 1996.

SANTOS, A. R. Metodologia científica: a construção do conhecimento. Rio de Janeiro: DP\&A, 2002.

SANTOS, C. M. B. N. Expatriadas nos Estados Unidos: desafios e conquistas. In: ENCONTRO ANUAL DA ASSOCIAÇÃO NACIONAL DE PÓS-GRADUAÇÃO E PESQUISA EM ADMINISTRAÇÃO, 27., 2003, Atibaia. Anais... Atibaia: EnANPAD, 2003. p. 1-16.

SAYÃO, A. C. M.; TANURE, B.; DUARTE, R. G. Mudanças na cultura pós-aquisição: o caso da empresa alfa. In: ENCONTRO ANUAL DA ASSOCIAÇÃO NACIONAL DE PÓS-GRADUAÇÃO E PESQUISA EM ADMINISTRAÇÃO, 30., 2006, Salvador. Anais... Salvador: EnANPAD, 2006. p. 1-16.

SILVA, M. As dimensões culturais da qualidade: um estudo em empresas ganhadoras do prêmio nacional da qualidade. In: ENCONTRO ANUAL DA ASSOCIAÇÃO NACIONAL DE PÓS-GRADUAÇÃO E PESQUISA EM ADMINISTRAÇÃO, 26., 2002, Salvador. Anais... Salvador: EnANPAD, 2002. p. 1-16.

SPINK, P. O lugar do lugar na análise organizacional. Revista de Administração Contemporânea, v. 5, p. 11-34, 2002. Edição Especial.

TANURE, B.; CANÇADO, V. L. Fusões e aquisições: aprendendo com a experiência brasileira. Revista de Administração de Empresas, v. 45, n. 2, p. 1-13, abr./jun. 2005a. 
Aquisições transnacionais entendendo os impactos da cultura local. In: ENCONTRO ANUAL DA ASSOCIAÇÃO NACIONAL DE PÓS-GRADUAÇÃO E PESQUISA EM ADMINISTRAÇÃO, 19., 2005b, Brasília. Anais... Brasília: EnANPAD, 2005b. p. 1-16.

VEIGA, L. F. A.; ROCHA, A. Escolha de mercados externos, distância cultural e comprometimento: estudo de casos de grandes empresas brasileiras. In: ENCONTRO ANUAL DA ASSOCIAÇÃO NACIONAL DE PÓS-GRADUAÇÃO E PESQUISA EM ADMINISTRAÇÃO, 15., 2001, Campinas. Anais... Campinas: EnANPAD, 2001. p. 1-16.

YIP, G. S. Globalização: como enfrentar desafios da competitividade mundial. São Paulo: SENAC, 1996.

Recebido: 26/09/2007 Received: 09/26/2007

Aprovado: $10 / 10 / 2007$ Approved: 10/10/2007 